\title{
Importancia económica y biológica de la alfalfa en el centro de Chihuahua
}

Economic and biological importance of alfalfa in Chihuahua center

\author{
Sandra Alvarado Ríos ${ }^{1}$, Sergio Guerrero Morales ${ }^{1 凶}$, José Álvaro Anchondo Nájera ${ }^{1}$, Bertha Alicia Rivas \\ Lucero $^{1}$, Abdón Palacios Monarrez ${ }^{1}$ \\ ${ }^{1}$ Universidad Autónoma de Chihuahua-Facultad de Ciencias Agrícolas y Forestales Km 2 1² Carretera Delicias- \\ Rosales C.P. 33000, Tel: 6394722726. \\ ${ }^{\square}$ Autor para correspondencia: sguerrer@uach.mx
}

\begin{abstract}
Recibido: $15 / 07 / 2017 \quad$ Aceptado: $10 / 12 / 2017$
\end{abstract}
\section{RESUMEN}

El cultivo de alfalfa en la región centro sur del estado de Chihuahua, es el de mayor superficie sembrada $(35,865.50$ ha.) y representa una fuente de ingresos para familias enteras. La región es de las principales abastecedoras de alfalfa de los estados de Coahuila y Durango. El presente estudio se realizó con los objetivos de: Describir la distribución del ingreso entre productores e intermediarios por venta de alfalfa. Analizar el significado hídrico de la exportación de alfalfa a otras regiones. Determinar la importancia del cultivo de alfalfa en el control biológico de plagas en el nogal. Se realizaron entrevistas semiestructuradas a productores, intermediarios de alfalfa, e investigadores. La información obtenida, se analizó estadísticamente con los programas de Excel y SAS. El precio de venta de alfalfa, al consumidor final oscila de entre \$2.5 y \$ 4.00 por kg de alfalfa henificada. La producción total de alfalfa en el 2015 fue de 609713.66 toneladas en base a peso seco. El 51\% de la producción se exporto a otros estados, que equivalen a $219496921 \mathrm{~m}^{3}$ de agua exportada. La venta del 51\% de alfalfa a otros estados genera un ingreso a la región de $\$ 971,731,200.00$ (con un 20\% de ganancia del intermediario) a $\$ 1$ 195, 976,923.00 (con un 35\% de ganancia del intermediario). Se encontró que la alfalfa es un hábitat muy importante de insectos que regulan a insectos plagas en el cultivo de nogal.

Palabras clave: Alfalfa, Producción, Ingreso, Control biológico.

\begin{abstract}
ABSTRAC
The cultivation of alfalfa in the south central region of the state of Chihuahua is the one with the largest area planted $(35,865.50$ ha.) And represents a source of income for whole families. The region is one of the main suppliers of alfalfa to the states of Coahuila and Durango. The present study was carried out with the objectives of: Describing the distribution of income between producers and intermediaries by sale of alfalfa. Analyze the hydric significance of the export of alfalfa to other regions. Determine the importance of alfalfa cultivation in the biological control of pests in walnut. Semistructured interviews were conducted with producers, alfalfa intermediaries, and researchers. The information obtained was analyzed statistically with Excel and SAS programs. The sale price of alfalfa, to the final consumer ranges

Revista Científica Biológico Agropecuaria Tuxpan 5 (2)


from $\$ 2.5$ to $\$ 4.00$ per $\mathrm{kg}$ of hay alfalfa. The total production of alfalfa in 2015 was 609713.66 tonnes based on dry weight. $51 \%$ of the production was exported to other states, equivalent to $219,496,921 \mathrm{~m} 3$ of water exported. The sale of $51 \%$ of alfalfa to other states generates an income to the region of $\$$ $971,731,200.00$ (with a $20 \%$ profit of the intermediary) to $\$ 1195,976,923.00$ (with a $35 \%$ profit of the intermediary). It was found that alfalfa is a very important habitat of insects that regulate insect pests in the walnut crop.

Keywords: Alfalfa, Production, Income, Biological control.

\section{INTRODUCCIÓN}

En México la actividad ornamental es una La alfalfa es uno de los cultivos de mayor superficie $(35,865.51$ hectáreas) e importancia en la región centro-sur del estado de Chihuahua, la cual comprende los municipios de: Julimes, Meoqui, Saucillo, Camargo, Delicias, San Francisco de Conchos, La Cruz y Rosales. El potencial promedio de producción es de 24 ton por hectárea de forraje seco al año, lo que contrasta con el promedio regional real que es de 17.69 ton $\mathrm{ha}^{-1}$, debido a que no se aplica el paquete tecnológico en su totalidad SIAP-SAGARPA (2015).

La alfalfa es un cultivo que brinda sustento económico a muchas familias chihuahuenses. La producción es para consumo regional y comercialización a otros estados (Durango, Coahuila y Zacatecas). Desarrollándose así una cadena de compra y distribución, donde los beneficios se distribuyen entre productores y comercializadores, siendo el consumidor final el que se encarga de fijar el precio. El rendimiento promedio de alfalfa en la región es de 17.69 ton ha-1, lo que contrasta con el potencial promedio de 24 ton por ha-1 de forraje seco al año, Lara y Jurado (2014). La superficie sembrada de alfalfa se ha incrementado en la región, porque una cantidad de la producción se exporta a otros estados. Un aspecto a considerar en la producción de alfalfa es el alto volumen de agua requerido, la cual es uno de los recursos escasos en la región (Rodríguez, 2014). El envío de alfalfa a otras regiones significa en los hechos una manera de exportar agua, un recurso cuya disponibilidad se reduce día con día en la región agrícola de Delicias.

El cultivo de alfalfa es asimismo un excelente hábitat para propagación de insectos que resulten ser benéficos para otros cultivos, Zaviezo (2004) y representa condiciones para un control natural de plagas Zumoffen, et al. (2010. La cantidad de predadores para pulgones son mayores en nogales establecidos en alfalfa, que, en nogales solos, Martínez (2002). Estos insectos son de gran importancia en el control de plagas del nogal.

\section{MATERIALES Y MÉTODOS}

El área de estudio se encuentra ubicada en la región centro sur de Chihuahua, que comprende los municipios de: Delicias, Meoqui, Camargo, San Francisco de Conchos, La Cruz, Saucillo, Julimes y Rosales. Estos municipios, cuentan con una superficie total de 3.65 millones de hectáreas, de las cuales más de 99 mil hectáreas se destinan a la agricultura y en su totalidad son de riego, programa sectorial-Chihuahua (20042010).

La investigación fue descriptiva, se determinó el tamaño de muestra de productores de alfalfa que fue de 167 productores. Se realizaron entrevistas semiestructuradas a productores e intermediarios de alfalfa, se obtuvieron datos de precios de compra, de venta, de producción, de variedades sembradas y destino de la producción. Asimismo, se visitó a 
diferentes investigadores, los cuales contaban con un gran historial de trabajo en los huertos de nogal, tanto en la región como en otros lugares, también especialistas en plagas e insectos benéficos, que proporcionaron información y estudios realizados basados en el cultivo de la alfalfa, huertos de nogal, insectos benéficos y plagas. Con base a esto se realizó un análisis estadístico con los programas de Excel y SAS,

Las principales fuentes de información utilizadas fueron:

Entrevistas semiestructuradas aplicadas a productores e intermediarios del cultivo de alfalfa. Entrevista directa con investigadores de la Facultad de Ciencias Agrícolas y Forestales y externos. Fuentes indirectas como: Publicaciones científicas, libros, documentos, etc.

Se analizaron de manera generalizada los costos de producción del cultivo de la alfalfa, tomando en cuenta el costo de los insumos y mecanismos necesarios para la producción del cultivo. Se determinó, de acuerdo a los requerimientos de agua del cultivo $\left(12,000 \mathrm{~m}^{3}\right.$ $\left.\mathrm{ha}^{-1}\right)$, y la producción total de alfalfa en verde de la región (3, 293, 924 ton.), la cantidad de agua que se está exportando a otras regiones del país. Se realizó una descripción del impacto biológico de la alfalfa en especies benéficas en el cultivo del nogal, en que momento están presentes y pueden ser utilizadas y la significancia y contribución de estos hacia la agricultura.

\section{RESULTADOS}

Se describe la cadena de distribución del ingreso económico por la venta de alfalfa entre productores, intermediarios y consumidor final, así como el significado hídrico de la exportación de alfalfa a otras regiones y una ponderación de los beneficios indirectos del cultivo de alfalfa, en la aportación de insectos benéficos a otros cultivos.
El precio de la alfalfa depende de la oferta y demanda, esto origina una variación de precios en el cultivo de alfalfa durante todo el año. Los meses en que tiene mayor valor el cultivo oscilan de marzo a mayo y de septiembre a noviembre (figura 1).

Los precios son fijados por el consumidor final, sin embargo, el intermediario realiza sus adecuaciones para concretar el precio al productor, que oscila de $\$ 2.5$ a $\$ 4.00 \mathrm{~kg}$ de acuerdo a la oferta y demanda.

La producción promedio de alfalfa henificada en la región es de 17 ton ha $^{-1}$, el costo de producción promedio de alfalfa bajo condiciones de riego según Lara y Jurado (2014) es de \$24 608 ha $^{-1}$, esta producción genera un ingreso de $\$ 42,500$ ha. Considerando el costo de producción y el precio promedio de venta de $\$ 2.5$ por $\mathrm{kg}$ de alfalfa, el agricultor obtiene en promedio una ganancia de $\$ 17,892 \mathrm{ha}^{-1}$.

De acuerdo con las entrevistas realizadas a productores e intermediarios y la información otorgada por el SIAP-SAGARPA (2015), la producción total en verde fue de 3,293, 923 toneladas que equivalen a 609, 713.67 toneladas de alfalfa en seco. De esta producción se encontró que el 51\% (310,954 ton), se exporta a los estados de Durango, Coahuila y Zacatecas.

La alfalfa que se queda en la región es por qué algunos productores cuentan con establos, ranchos ganaderos o engordas con algún tipo de ganado (bovino, porcino, caprino, ovino) o por qué su venta se realiza a ganaderos locales. La alfalfa que se produce para autoconsumo es la de menor porcentaje $19 \%$, la venta parcial ocupa un segundo lugar con un porcentaje del $30 \%$, resultando mayor negocio la venta total de alfalfa. La venta del $51 \%$ de la producción de alfalfa, a un precio promedio de $\$ 2.5 \mathrm{~kg}$. genero un ingreso a los productores de la región centro 
sur de Chihuahua de $\$ 777,383,000.00$. En relación al intermediario, de acuerdo a la información recabada, este vende la alfalfa a un precio de un $20 \%$ al $35 \%$ arriba de como se le paga al productor, lo que equivale a una ganancia de $\$ 0.625$ a $\$ 1.346$ por $\mathrm{kg}$ de alfalfa henificada vendida.

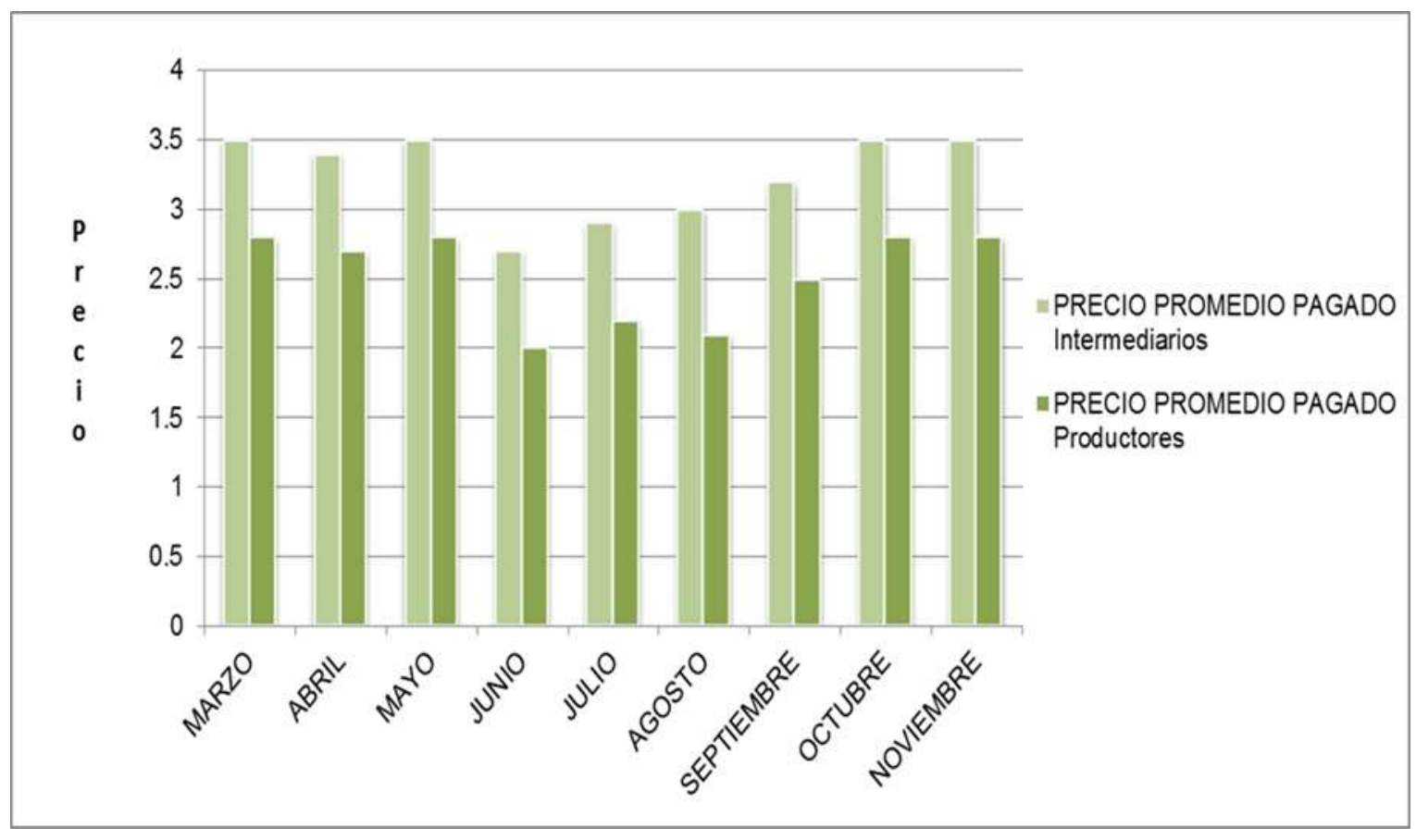

Figura 1. Precios de la alfalfa prevalentes durante el año 2015. Estudio de caso, importancia económica y biológica de la alfalfa en la región centro-sur del estado de Chihuahua. FCAyFUACH. 2017.

Es decir, el intermediario obtiene en promedio una ganancia por la venta de las 310,954 toneladas de alfalfa henificada de \$194,346 200 al ganar el 20\%, y de 418, 591, 923 cuando gana el $35 \%$.

Considerando lo anterior, el ingreso a la región por la venta del $51 \%$ de la producción de alfalfa (310, 954 ton de alfalfa henificada) es de $\$ 971$, $731,250.00$ (con un $20 \%$ de ganancia del intermediario) a $\$ 1$ 195, 976,923.00 (con un $35 \%$ de ganancia del intermediario).
Análisis Hídrico Realizado con la Exportación de Alfalfa a Otras Regiones

El consumo de agua que se requiere para la superficie total sembrada (35,865.51 ha) en la región es de 430, 386,120 $\mathrm{m}^{3}$. Considerando que para producir 17 ton ha ${ }^{-1}$ de alfalfa henificada se requieren $12,000 \mathrm{~m}^{3} \mathrm{ha}^{-1}$. La producción de alfalfa henificada en la región en el 2015 fue de 609,714 ton, de esta cantidad el 51\% (310,954 ton) se exportó a los estados mencionados anteriormente. Lo anterior corresponde a un volumen de agua de 219,496 $\mathrm{m}^{3}$ exportada en la venta de alfalfa Cuadro 1. 
Cuadro 1. Producción total de alfalfa y volumen de agua exportada por venta de Alfalfa. En estudio de caso, importancia económica y biológica de la alfalfa en la región centro-sur del estado de Chihuahua. FCAyF-UACH. 2017

\section{Total Producción $\quad 3,293,923$ Ton.}

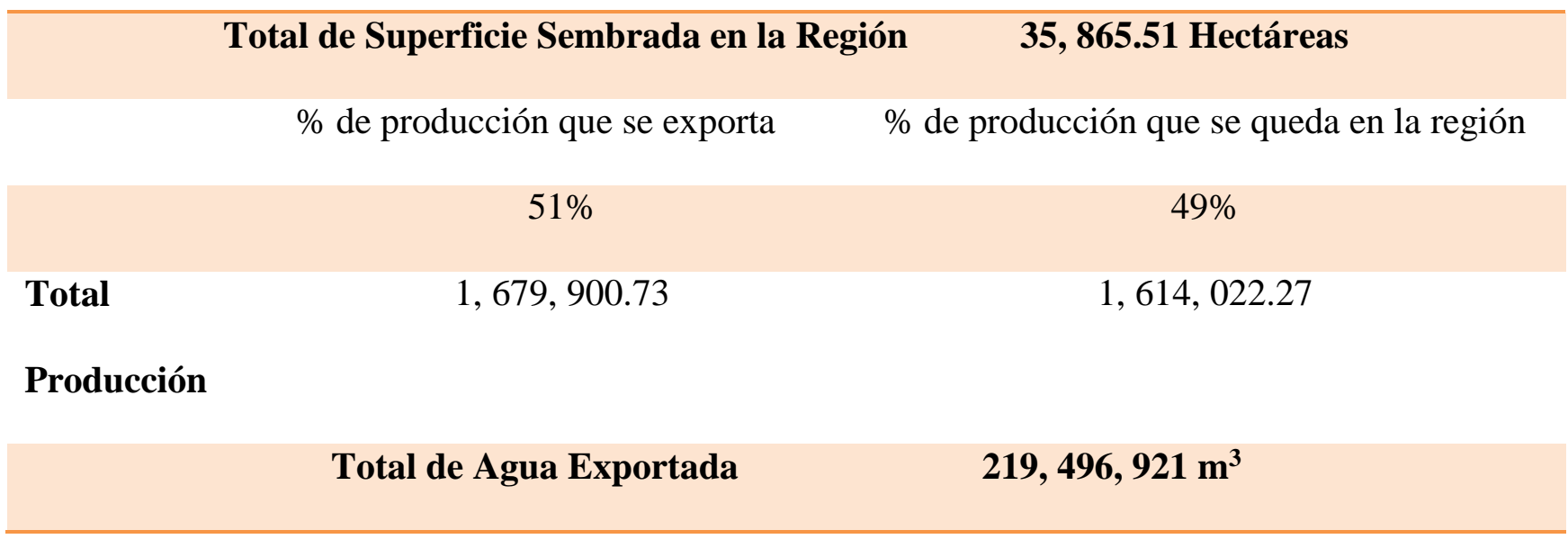

Es importante resaltar que la exportación de agua $\left(219,496,921 \mathrm{~m}^{3}\right)$ también deja una derrama económica para la región, por la venta de alfalfa la cual asciende a $\$ 971,713,250.00$ cuando el intermediario gana el $20 \%$ a $\$ 1,195,976,923.00$ cuando el intermediario gana $30 \%$. Considerando lo anterior por cada millar de agua exportada como alfalfa, la región recibe de $\$ 1,000.00$ a $\$ 5,448.00$ de acuerdo al precio de venta al consumidor final.

\section{Control Biológico de la Alfalfa en el Cultivo del Nogal}

Los campos de alfalfa dan alimento y/o refugio a una gran diversidad de polinizadores y enemigos naturales (parasitoides, predadores y entomopatógenos) que actúan como reguladores de las poblaciones de insectos dañinos, no sólo de la alfalfa sino de otros cultivos vecinos.

Los insectos benéficos provenientes del cultivo de alfalfa que se encontraron en huertos de nogal ayudaron de manera apreciable a controlar las plagas de nogal. Los nogales donde se encontró mayor índice de insectos benéficos, se observó que se encontraba situada una parcela de alfalfa a sus alrededores o no muy lejana (distancia menor a $300 \mathrm{~m}$ ). Es importante mencionar que en los meses donde se observó mayor índice de insectos fueron los meses con temperaturas más cálidas (Mayo - Agosto).

\section{CONCLUSIONES}

En base a la información obtenida el cultivo de alfalfa ocupa aproximadamente un $30 \%$ de la superficie total del distrito de riego 005 $(35,865.51$ ha $)$.

Existe variabilidad (de 2.5 a 4.0 $\mathrm{kg}$ ) en el precio de compra de alfalfa al productor durante el tiempo de cosecha, el mejor precio de venta es de marzo a mayo y de octubre a noviembre. 
Considerando el costo de producción y el precio promedio de venta de $\$ 2.5$ por $\mathrm{kg}$ de alfalfa, el agricultor obtiene en promedio una ganancia de $\$ 17,892 \mathrm{ha}^{-1}$.

De la Alfalfa cosechada en la región $(609,714$ toneladas de alfalfa henificada). El 51\% (310, 954 ton) se exporta a Durango, Coahuila y Zacatecas, principalmente. el 49\% (298 759.69) se queda en la región para usarse en establos locales.

El ingreso a la región por la venta del $51 \%$ de la producción de alfalfa (310, 954 ton de alfalfa henificada) es de $\$ 971,731,250.00$, con una ganancia para el intermediario de \$194,346 200 al ganar el $20 \%$, y de $\$ 1195,976,923$ con una ganancia al intermediario de 418, 591, 923 cuando gana el 35\%.

El consumo de agua para la producción de alfalfa en la región es de $430,386,120 \mathrm{~m}^{3}$ de esta cantidad con la venta de alfalfa fuera del estado se exporta $219,496,921 \mathrm{~m}^{3}$ de agua a otros estados.

La alfalfa es un excelente cultivo como hábitat para insectos que controlan plagas en el cultivo de Nogal.

\section{LITERATURA CITADA}

Alatorre, R.R., Bravo, M.H., Leyva, V.J. \& Huerta, P.A. (2010). Manejo integrado de plagas. Secretaría de Agricultura, Ganadería, Desarrollo Rural, Pesca y Alimentación. Subsecretaría de Desarrollo Rural. Dirección General e Apoyos para el Desarrollo. Recuperado de: www.sagarpa.gob.mx/desarrolloRural/Docu ments/fichasaapt/Manejo\%20integrado\%20de $\% 20 \mathrm{p}$ lagas.pdf

Anchondo, N.A. (2009). Producción agrícola en zonas áridas. Chihuahua, México Universidad autónoma de Chihuahua.

Charles, G.S., Larry, D.G. \& Erick, T.N. (2007). Managing insect in alfalfa, University of
California. Division of agriculture and natural resources. Publication 8295. Recuperado de: http://alfalfa.ucdavis.edu/IrrigatedAlfalfa/pd fs/UCAlfalfa8295Insects_free.pdf

Lara, M.C.R., Jurado G. P. 2014. Paquete tecnológico para producir alfalfa en el estado de chihuahua. Folleto técnico núm 52. INIFAP. Sitio Experimental La Campana Aldama, Chihuahua.

Martínez A., I. P. 2002. Fluctuación poblacional de pulgones y sus depredadores en huertas de nogal pecanero (Carya illinoensis Koch), con $\mathrm{y}$ sin alfalfa como cobertera vegetal en la Comarca Lagunera. Tesis de Maestría en Ciencias. FAZ-UJED, División de Estudios de Posgrado. Venecia, Durango, México. 172 p.

Montemayor, T.J., Aguirre, A.H., Olague, R.J., López, A.R., Rivera, G.M., Preciado, R.P., Montemayor I.R., Trejo, M.A., Segura, C., Orozco, V.J. \& Yescas, C.P. (2010). Uso de agua en la alfalfa (Medicago Sativa L.) con riego por goteo superficial. México, D.F., Revista Mexicana de Ciencias Pecuarias.

Oropeza, M.H. (2008). La Agricultura: Economía, Administración y Costos. México, D.F. Editorial Trillas.

Rodríguez, J. M. \& Aldaba, J. L. El sector agropecuario en el centro - sur de Chihuahua, México. Recuperado de: http://revistas.bancomext.gob.mx/rce/magazi nes/7315/RCE5.pdf

Rodríguez, M.C., (2014). Metodología de la siembra del cultivo de alfalfa (Medicago Sativa L.) en la región centro-sur del estado de Chihuahua. Universidad Autónoma de Chihuahua, Cd. Delicias, Chihuahua.

SAGARPA 2014. Resumen nacional por cultivo. Consultado el 13 de noviembre del 2015. Recuperado de: http://www.siap.gob.mx/agrresumennacional-por-cultivo/

Secretaria de Hacienda y Crédito Público. Financiera Nacional de Desarrollo Agropecuario, Rural, Forestal y Pesquero. (2014). Panorama de la alfalfa. Recuperado de: http://www.financierarural.gob.mx/informaci onsectorrural/panoramas/panorama\%20Alfalf a\%20(abr\%202014).pdf 
Zumoffen, L.; Salto, C. y Singorini, M. 2010. Alfalfa

(Medicago Sativa L.) como reservorio de insectos entomófagos. Revista FAVE Ciencias Agrarias 9 (1-2).

Copyright (c) 2017 Sandra Alvarado Rios, Sergio Guerrero Morales, José Álvaro Anchondo Nájera,

Bertha Alicia Rivas Lucero y Abdón Palacios Monarrez

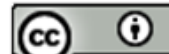

Este tex to está protegido por una licencia licencia Creative Commons 4.0

\begin{abstract}
Usted es libre para Compartir —copiar y redistribuir el material en cualquier medio o form ato-y Adaptar el documento —remezdar, transformar y crear a partir del material- para cualquier propósito, incluso para fines comerciales, siempre que cumpla la condición de:

Atribución: Usted debe dar crédito a la obra original de manera adecuada, proporcionar un enlace a la licencia, e in đicar si se han realizado cam bios. Puede hacerlo en cualquier form a razonable, pero no de form a tal que sugiera que tiene el apoyo del licenciante o 10 recibe por el uso que hace de la obra.

Resumendelicencia - Textocompletodelalicencia
\end{abstract}

